\title{
Holographic-Based mmW-Wideband Bidirectional Frequency Scanning Leaky Wave Antenna
}

\author{
Ali Araghi, Mohsen Khalily, Pei Xiao, Rahim Tafazolli \\ Institute for Communication Systems, Home of 5G Innovation Centre, University of Surrey, Guildford, UK \\ \{a.araghi, m.khalily, p.xiao, r.tafazolli\}@ surrey.ac.uk
}

\begin{abstract}
Utilizing the holography theory, a bidirectional wideband leaky wave antenna in the millimetre wave $(\mathrm{mmW})$ band is presented. The antenna includes a printed pattern of continuous metallic strips on an Alumina $99.5 \%$ sheet, and a surface wave launcher (SWL) to produce the initial reference waves on the substrate. To achieve a bidirectional radiation pattern, the fundamental TE mode is excited by applying a Vivaldi antenna (as the SWL). The proposed holographic-based leaky wave antenna (HLWA) is fabricated and tested and the measured results are aligned with the simulated ones. The antenna has $22.6 \%$ fractional bandwidth with respect to the central frequency of $30 \mathrm{GHz}$. The interference pattern is designed to generate a $15 \mathrm{deg}$ backward tilted bidirectional radiation pattern with respect to the normal of the hologram sheet. The frequency scanning property of the designed HLWA is also investigated.
\end{abstract}

Index Terms-leaky wave antennas, holography theory, surface waves, frequency-scanning, millimetre wave.

\section{INTRODUCTION}

Leaky wave antennas (LWAs) are considered as a promising candidate to be applied in the millimetre-wave $(\mathrm{mmW})$ band due to their high-gain and relatively simple feeding network with the inherent frequency-scanning capability. Their radiation mechanism is based on the leakage rate from a surface wave (SW) bounded in a medium. This leakage ends up with an electromagnetic aperture that defines the radiation characteristics such as direction of the beam and gain. As a result, the LWA implementation is nothing but designing an appropriate aperture from the structure in hand and to excite the aimed SW on it toward the direction of interest by applying a proper feed line commonly known as the surface wave launcher (SWL).

According to the velocity of waves in a guided medium that is higher or less than the speed of light, fast waves and slow waves are defined respectively. Fast waves are can lead to an "improper" leakage [1] of the SW, which is required to meet the propagation condition. To achieve an LWA from a substrate that supports slow waves, a periodic pattern of scatterers is needed to generate the space harmonics which can be analysed by the Floquet theorem [2]. The resulted radiating structure is the so-called periodic LWA (PLWA).

In the traditional PLWAs, the repeating distance of scatterers is controlled in a way that one (or more) of the space harmonics are located in the fast-wave region which ultimately defines the direction of beam. However, it is possible to deal with LWA design from an opposite angle, i.e. by first considering the direction of desired beam and then, defining the scatterer pattern based on that information.

Holography theory, originating from the optics, relies on the direction of excited SW on the substrate and the direction of aimed radiating beam to calculate the hologram pattern on the supporting substrate [3]. The initial SW is "reference wave" $\left(\vec{E}_{\text {ref }}\right)$, and the aimed radiating waves toward the direction of interest is obtained by defining an "object wave" $\left(\vec{E}_{o b j}\right)$ from a source located at the infinite incidents toward the sheet. The calculated scatterer pattern on the substrate represents the holographic leaky wave antenna (HLWA). By interfering the phase lines of $\vec{E}_{\text {ref }}$ with the map of $\vec{E}_{o b j}$ on the supporting substrate, a pattern of phase lines is obtained. It is possible to sample these phase lines by printing a set of metallic scatterers on the substrate based on the interferogram pattern. The reported variations of scatterers can be divided in three: continuous metal strips [4], [5], [6], hertzian dipoles [7], [8], [9], and the metasurfaces [10], [11], [12], [13]. Among these options, the third one has attracted lots of attention due to accurate sampling, control on polarization, and the inherent flexibility it offers. However, continuous metal strips still play an important role in the design of modern HLWAs [3], [6]. Comparing with the other two, their immediate advantages is the simplicity of calculation while the resulting performance in terms of achieved gain and the accuracy of pointed direction is similar to or even better than metasurface and dipole scatterers.

As a travelling SW on the substrate, the $\vec{E}_{\text {ref }}$ is in two general forms of TE and TM modes. A substrate without the metallized ground plane supports TE waves while the one with ground plane is suitable for TM propagation. It is shown that all modes are in a hybrid form [10], but to have an almost pure mode on the substrate, the first step is to select an appropriate SWL to excite the interested mode. Vivaldi antennas are wellknown to generate TE waves while the monopole antennas end up in TM mode on the substrate; other types of SWLs are reported in [3], [6], [13]. TE reference waves result in bidirectional radiation from the HLWA in hand owing to the absence of the ground plane. As an example, recently [14] proposes a metasurface-based HLWA with bidirectional radiation pattern in the microwave band. By applying continuous metallic strips, [15] presents a bidirectional broadside HLWA, and [16] provides the electromagnetic modelling of that, both fed by a bulky horn antenna as the SWL.

In this paper, a fully printed wideband bidirectional HLWA in the $\mathrm{mmW}$ band by utilizing of the continuous metal strips 
is presented for the first time and its frequency-scanning capability is studied.

\section{HOLOGRAPHIC-BASED LWA DESIGN}

\section{A. Initial Considerations}

The hologram pattern is obtained considering the influence of both reference and object waves on the sheet. The manipulation process is divided into two steps of "recording" and the "reconstruction". The first step is to capture the interference pattern of $\vec{E}_{r e f}$ and $\vec{E}_{o b j}$. The second step is to apply a SWL on the substrate in order to generate $\vec{E}_{\text {ref }}$ on the calculated hologram sheet to reconstruct $\vec{E}_{o b j}$.

It is shown in [3], [6] that to excite the SW on a dielectric slab, the substrate permittivity should be high enough in a way that propagating waves properly latched into the substrate. In addition, the dielectric loss should be as low as possible to result in appropriate radiation efficiency. These issues become more important for mmW-HLWA design since directly effect on the aperture illumination. As a result, Alumina $99.5 \%$ is chosen as the supporting substrate with $\epsilon_{r}=9.9$ and extremely low $\tan \delta$. The substrate thickness is also preferred to be larger than $\lambda_{r e f} / 10$ with $\lambda_{r e f}$ as the effective wavelength of the excited $\vec{E}_{\text {ref }}$.

The design frequency of operation is chosen to be $f=$ $30 \mathrm{GHz}$ as a promising band to be allocated in the fifthgeneration $(5 \mathrm{G})$ networks for delivering point to point connections like wireless backhauling [17] that requires highgain antennas with directional radiation pattern. Based on the selected frequency and the above-mentioned criteria, the substrate thickness is considered $h=0.254 \mathrm{~mm}$. It must be noted that, dielectric constant and thickness of the substrate would define the effective $\lambda_{\text {ref }}$ that should be taken into consideration throughout the recording process.

\section{B. Recording Process}

The hologram sheet size is chosen to be $80 \times 90 \mathrm{~mm}^{2}$, large enough to contain number of reference wavelengths, placed on the $x-y$ plane. Thanks to the ignorable $\tan \delta$ of the Alumina, radial reference waves from an ideal feed located at the origin with phase constant of $\beta_{r}=2 \pi / \lambda_{\text {ref }}$, is described as follows;

$$
\vec{E}_{r e f}=A e^{-j \beta_{r} r}
$$

where $r=\sqrt{x^{2}+y^{2}}$ and $A$ is the wave amplitude.

Regarding an object wave from the open space toward the sheet aligned at the angle $\left(\theta_{m}, \phi_{m}\right)$ in a standard right-hand co-ordination system, the map of $\vec{E}_{o b j}$ phase lines on the sheet is obtained using the following equation.

$$
\vec{E}_{o b j(x, y)}=B e^{j k_{0}\left\{\sin \left(\theta_{m}\right) \cos \left(\phi_{m}\right) x+\sin \left(\theta_{m}\right) \sin \left(\phi_{m}\right) y\right\}},
$$

where $B$ is the amplitude and $k_{0}$ is the wave vector of the object waves.

The object waves is chosen to be directed toward $\left(\theta_{m}=\right.$ $15 \mathrm{deg}, \phi_{m}=270 \mathrm{deg}$ ) at the operating frequency of $30 \mathrm{GHz}$. Under this circumstance, the superposition of (1) and (2) lead

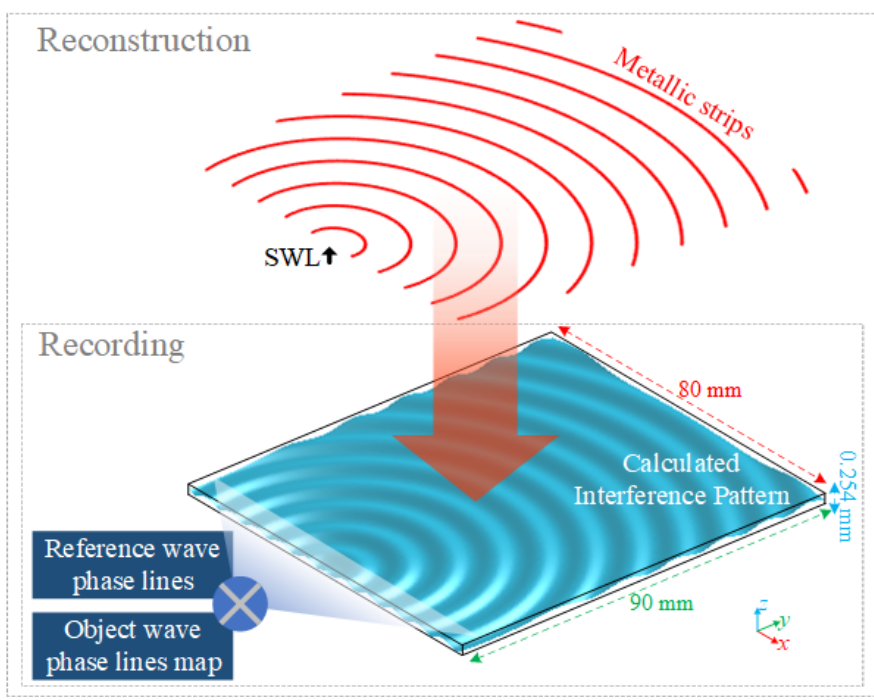

Fig. 1. The "Recording" and "Reconstruction" process of calculating the hologram sheet for an HLWA with radiation beam toward $\left(\theta_{m}=15 \mathrm{deg}, \phi_{m}=\right.$ $270 \mathrm{deg})$.

to the hologram pattern. The "Recording" process is illustrated in Fig. 1.

\section{Reconstruction Process}

To build the calculated hologram on the substrate, metallic strips are applied. These strips shorten the SW's E-field lines at their specific position, providing the roots of the interference pattern. As a result, the manipulated hologram will possess an interference pattern with reduced phase information. Utilizing this approach, the remaining phase information is lost. However, the sampled E-fields are quite enough to make the radiation pattern accurately pointed to the direction of interest.

Hence, printing the metallic continuous strips at the local maxima of calculated interference phase lines on the dielectric slab, accompanied by designing an appropriate SWL to generate the initial SWs is the "Reconstruction" process shown in Fig. 1.

\section{Hologram Realization}

In order to form a bidirectional radiation from the sheet, the reference wave should be a TE mode SW, therefore a Vivaldi antenna is designed as the SWL. The flares-curve are shaped in a way that the antenna operates at the central frequency of $30 \mathrm{GHz}$ with maximum possible bandwith in the structure including the hologram scatterers as well. The designed HLWA based on the calculated interference pattern of Fig. 1 is presented in Fig. 2. The parameter size are $w_{f}=$ $0.245 \mathrm{~mm}, g_{f}=3.75 \mathrm{~mm}, p_{f}=1.75 \mathrm{~mm}, q_{f}=1.41 \mathrm{~mm}$, $f_{f}=4.79 \mathrm{~mm}, w_{s}=2 \mathrm{~mm}, l_{H}=17 \mathrm{~mm}$. Among these parameters, the last two will severely effect the constructed beam, need to be explored more.

Comparing to the initial sheet size of Fig. 1, the hologram dimensions is increased toward the $-\hat{a}_{y}$ by the value of $l_{H}$ (see Fig. 2). This extra space result in proper response of the hologram to the scattered waves from the metallic strips 


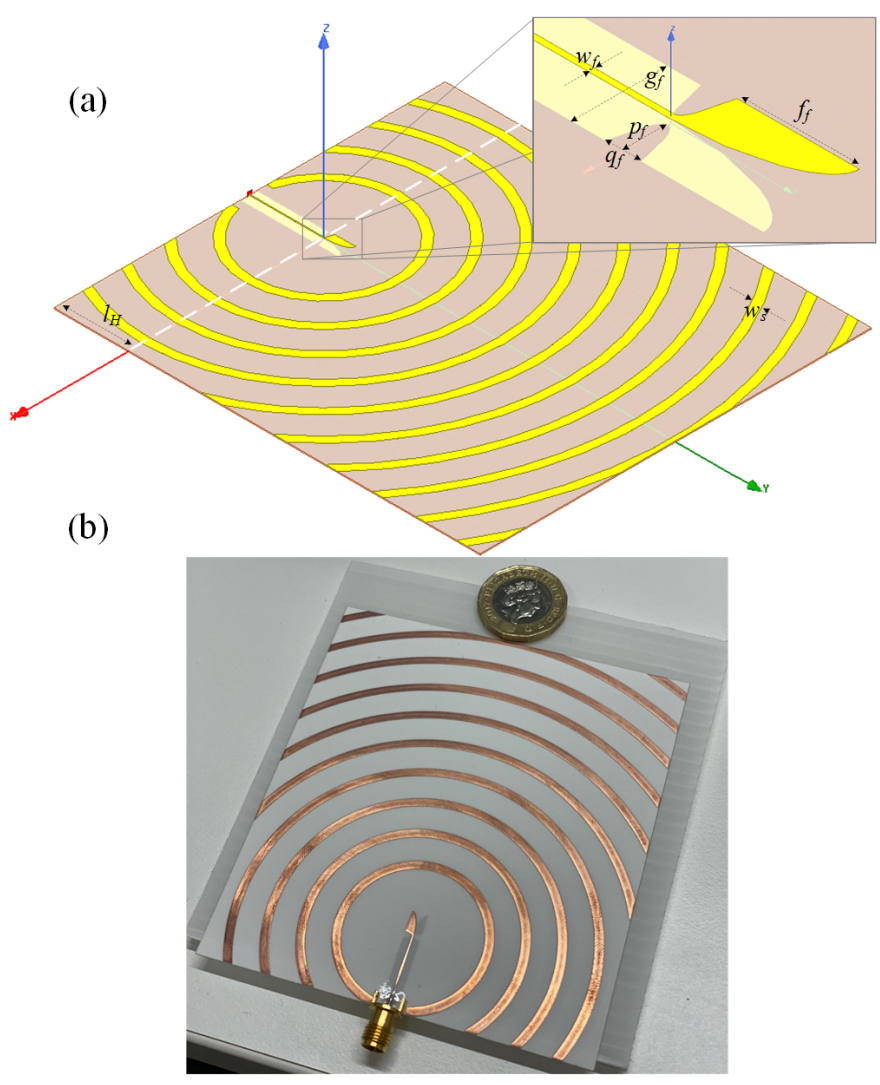

Fig. 2. The proposed HLWA based on the calculated interference pattern. (a) schematic view, (b) fabricated.

located in front of the SWL. Without this expansion, the hologram would have unwanted beams around the $x-$ axis. The other important parameter is the width of the metallic strips $\left(w_{s}\right)$. It is shown in [6] that the strip's width has a direct influence on the reference wave number $\left(\lambda_{r e f}\right)$. In order to accurately point the beam towards the targeted direction, this effect must be considered prior to the design process. Without this consideration, the final radiation pattern would vary by several degrees comparing to the initial theoretical value. As a result, the optimal value of strip widths is obtained as $w_{s}=2 \mathrm{~mm}$ to have the proper leakage.

Finally, it must be noted that to embody the physical structure of the designed HLWA, the closest metallic strip to the SWL shown in Fig. 1 is eliminated, providing the room for the Vivaldi placement. As a result, the number of strips in Fig. 2 is one less than that of Fig. 1.

\section{RESUlTS AND Discussions}

\section{A. Leakage Performance and the Reflection Coefficient}

The bidirectional E-field leakage from the proposed HLWA at the operating frequency of $f=30 \mathrm{GHz}$ is simulated by ANSYS HFSS and presented in Fig. 3. As it is clear from the figure, as long as the generated SWs surf the substrate, an "improper" leakage is shaped toward the direction of interest.

The SWL is designed in a way that the maximum possible bandwidth for this hologram is achieved. The simulated (CST

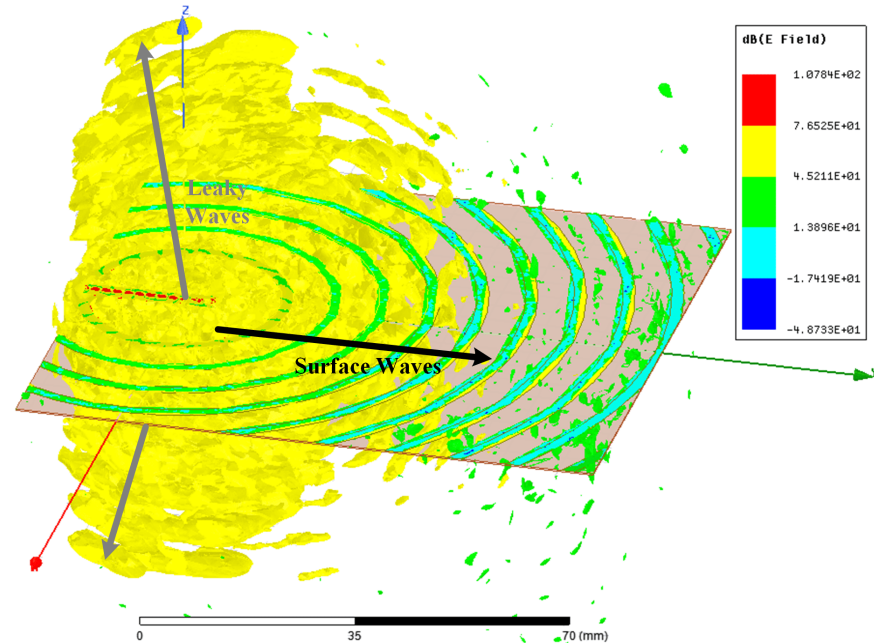

Fig. 3. The mechanism of bidirectional leakage toward the direction of interest from the designed HLWA at $f=30 \mathrm{GHz}$.

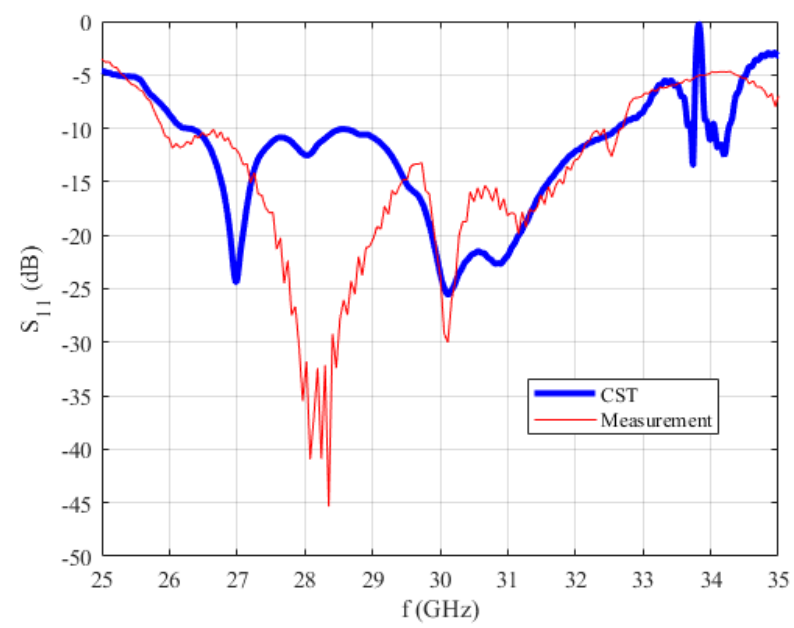

Fig. 4. The simulated and measured S-parameter.

MWS) and measured S-parameters are shown in Fig. 4. This figure indicates $22.6 \%(21.3 \%)$ measured (simulated) fractional bandwidth from $25.9 \mathrm{GHz}$ to $32.7 \mathrm{GHz}(26.2 \mathrm{GHz}$ to $32.6 \mathrm{GHz}$ ) with respect to the central frequency of $30 \mathrm{GHz}$ expressing the wide-band performance of the antenna.

\section{B. Radiation Characteristics}

The simulated radiation patterns of directivity at different frequencies of the operating bandwidth are illustrated in Fig. 5.

This figure shows that the designed hologram sheet has an accurate response upon the SWL, properly directs the final beam to the aimed angle of $\theta_{m}=15 \mathrm{deg}, \phi_{m}=270 \mathrm{deg}$ (and its directional mirror against the $\theta=90 \mathrm{deg}$ axis) at $f=30 \mathrm{GHz}$. The frequency scanning is also observable from the figure with a main beam point ranging from $\theta_{m}=30(150) \mathrm{deg}$ at $f=26.5 \mathrm{GHz}$ to $\theta_{m}=6(174) \mathrm{deg}$ at $f=32.5 \mathrm{GHz}$. According to this figure, the constructed beam 


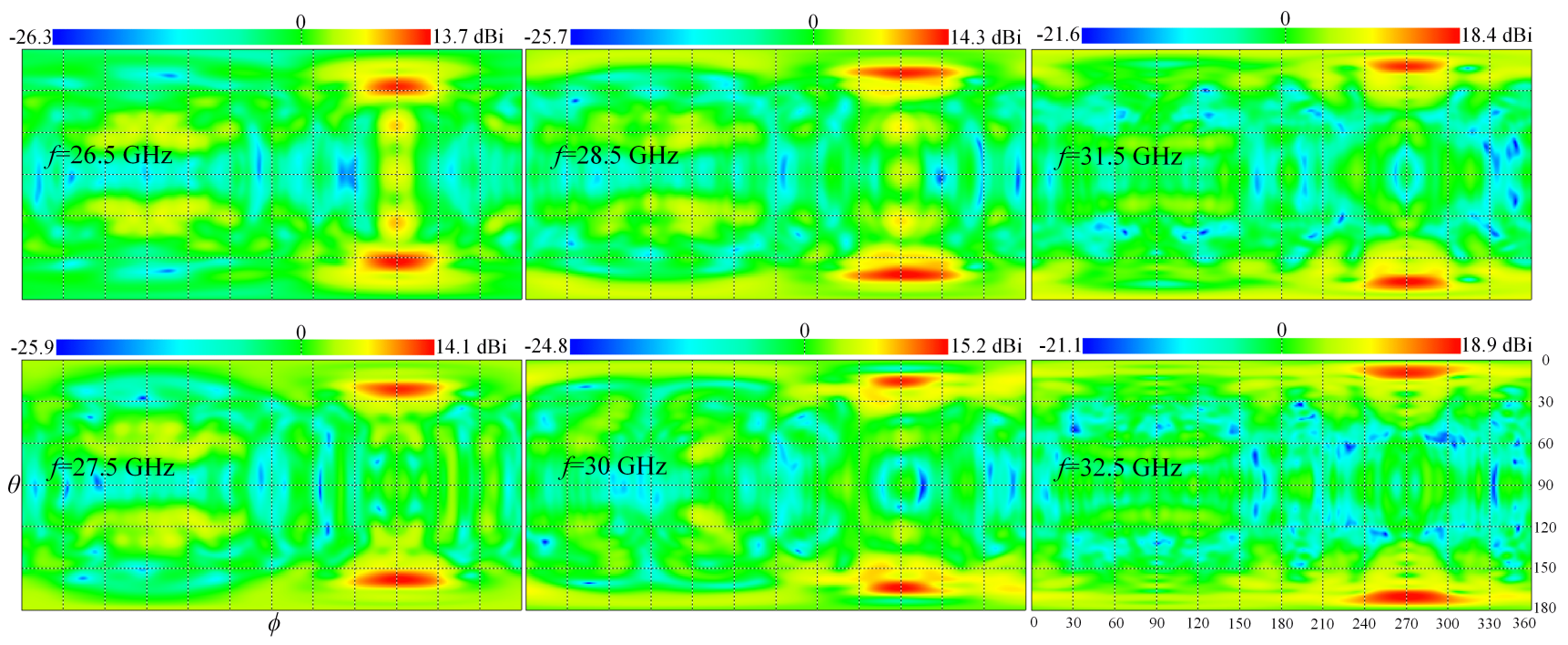

Fig. 5. The constructed bidirectional radiation pattern of the directivity at different frequencies.

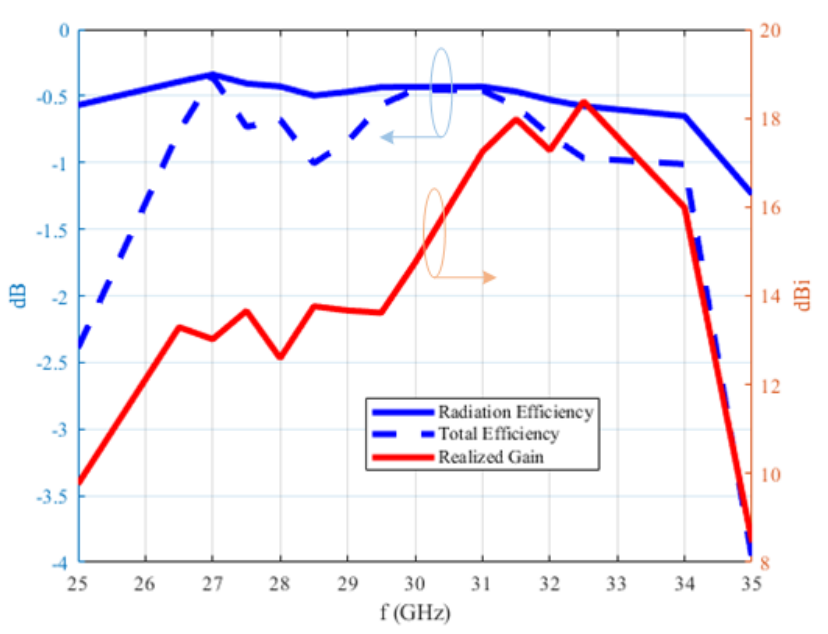

Fig. 6. The radiation/ total efficiencies and the realized gain versus frequency.

is relatively narrower across the $\theta$ angles than $\phi$. This issue can be described in this way: the feed is launching SWs along the $y$ - axis more powerfully than along the $x$. Consequently, the generated aperture area is distributed more effective along the $y-$ axis, leading to a narrower beam toward the $\theta$ direction.

The radiation efficiency, total efficiency, and the realized gain are shown in Fig. 6. The maximum realized gain of the designed HLWA is $18.4 \mathrm{dBi}$ occurred at $32.5 \mathrm{GHz}$.

\section{CONCLUSion}

A wideband bidirectional holographic-based leaky wave antenna was introduced in this paper. With the structure lying on $x-y$ plane, the interference pattern was designed to have two tilted beams at $30 \mathrm{GHz}$; one toward $\theta=15 \mathrm{deg}$, $\phi=270 \mathrm{deg}$ and the other to $\theta=165 \mathrm{deg}, \phi=270 \mathrm{deg}$. Thanks to the wideband performance of the designed surface wave launcher, the calculated hologram has more than $6 \mathrm{GHz}$ bandwidth around the operating frequency. The beam direction varies $24 \mathrm{deg}$ at both directional sides while the frequency changes from $26.5 \mathrm{GHz}$ to $32.5 \mathrm{GHz}$ where the maximum realized gain of the designed antenna is achieved $(18.4 \mathrm{dBi})$.

\section{REFERENCES}

[1] D. R. Jackson, A. A. Oliner, Leaky-wave antennas, Modern antenna handbook, pp. 325-367, 2008

[2] J. H. Choi, T. Itoh, Beam-scanning leaky-wave antennas, Handbook of antenna technologies, Springer, pp. 1697-1735, 2016.

[3] C. Rusch, Holographic antennas, Handbook of antenna technologies, Springer, pp. 2689-2725, 2016.

[4] C. Rusch, S. Beer, P. Pahl, and T. Zwick, "Multilayer holographic antenna with beam scanning in two dimensions at W-band," 2013 7th European Conference on Antennas and Propagation (EuCAP). IEEE, pp. 26252628, 2013

[5] C. Rusch, J. Schäfer, H. Guian, and T. Zwick, "2D-scanning holographic antenna system with Rotman-lens at $60 \mathrm{GHz}$," 8th European Conference on Antennas and Propagation (EuCAP 2014),IEEE, pp. 196-199, 2014.

[6] C. Rusch, J. Schäfer, H. Guian, P.Pahl, and T. Zwick, "Holographic mmW-Antennas With $T E_{0}$ and $T M_{0}$ Surface Wave Launchers for Frequency-Scanning FMCW-Radars", IEEE Transactions on Antennas and Propagation, vol. 63, no. 4, pp. 1603-1613, 2015.

[7] M. ElSherbiny, A. E. Fathy, A. Rosen, G. Ayers, and S. M. Perlow, "Holographic antenna concept, analysis, and parameters," IEEE Transactions on Antennas and Propagation, vol. 52, no. 3, pp. 830-839, 2004.

[8] A. Sutinjo, M. Okoniewski, and R. H. Johnston, "A holographic antenna approach for surface wave control in microstrip antenna applications," IEEE Transactions on Antennas and Propagation, vol. 58, no. 3, pp. 675682, 2010.

[9] A. Sutinjo, M. Okoniewski, "A surface wave holographic antenna for broadside radiation excited by a traveling wave patch array," IEEE Transactions on Antennas and Propagation, vol. 59, no. 1, pp. 297-300, 2010.

[10] B. H. Fong, J. S. Colburn, J. J. Ottusch, J. L. Visher, and D. F. Sievenpiper, "Scalar and tensor holographic artificial impedance surfaces," IEEE Transactions on Antennas and Propagation, vol. 58, no. 10, pp. 32123221, 2010.

[11] M. Karimipour, N. Komjani, "Realization of Multiple Concurrent Beams With Independent Circular Polarizations by Holographic Reflectarray," IEEE Transactions on Antennas and Propagation, vol. 66, no. 9, pp. 46274640, 2018. 
[12] M. Karimipour, N. Komjani, "Holographic-inspired multibeam reflectarray with linear polarization," IEEE Transactions on Antennas and Propagation, vol. 66, no. 6, pp. 2870-2882, 2018.

[13] H. Oraizi, A. Amini, A. Abdolali, and A. M. Karimimehr, "Design of Wideband Leaky-Wave Antenna Using Sinusoidally Modulated Impedance Surface Based on the Holography Theory," IEEE Antennas and Wireless Propagation Letters, vol. 17, no. 10, pp. 1807-1811, 2018.

[14] M. Movahhedi, M. Karimipour, and N. Komjani, "Multibeam Bidirectional Wideband/ Wide-Scanning-Angle Holographic Leaky-Wave Antenna," IEEE Antennas and Wireless Propagation Letters, vol. 18, no. 7, pp. 1507-1511, 2019.

[15] T. Quach, D. McNamara, and A. Petosa, "Holographic antenna realised using interference patterns determined in presence of dielectric substrate,' Electronics Letters, vol. 41, no. 13, pp. 724-725, 2005.

[16] P. Sooriyadevan, D. McNamara, A. Petosa, and A. Ittipiboon, "Electromagnetic modelling and optimisation of a planar holographic antenna,' IET Microwaves, Antennas, and Propagation, vol. 1, no. 3, pp. 693-699, 2007.

[17] R. Lombardi, "Microwave and Millimetre-wave for 5G Transport," ETSI White Paper, no. 25, 2018. 